\title{
Relationship between miR-7 expression and treatment outcomes with gefitinib in non-small cell lung cancer
}

\author{
KUN MOU ${ }^{1}$, WEIWEI GU ${ }^{1}$, CUIHUA GU ${ }^{1}$, JINZHONG ZHANG ${ }^{1}$, \\ WENJIE QWANG ${ }^{1}$, GANG REN ${ }^{1}$ and JING TIAN ${ }^{2}$ \\ ${ }^{1}$ Department of Oncology, People's Hospital of Laiwu City, Laiwu Hospital Affiliated to Taishan Medical \\ College, Laiwu, Shandong 271100; ${ }^{2}$ Department of Internal Medicine, Shandong Cancer Hospital Affiliated \\ to Shandong University, Shandong Academy of Medical Sciences, Jinan, Shandong 250117, P.R. China
}

Received July 5, 2016; Accepted September 29, 2016

DOI: $10.3892 / \mathrm{ol} .2016 .5290$

\begin{abstract}
The aim of the present study was to assess the effects of gefitinib chemotherapy on the serum levels of miR-7 in patients with non-small cell lung cancer (NSCLC). A total of 126 patients were enrolled in the present study (stage I-II, $\mathrm{n}=54$ and stage III-IV, $\mathrm{n}=72$ ). Patients with stage I-II NSCLC underwent surgery in combination with gefitinib chemotherapy, whereas only gefitinib chemotherapy was administered to patients with stage III-IV disease. Serum levels of miR-7 before and after treatment were measured with quantitative polymerase chain reaction using fluorogenic probes, and miR-7 positivity and scoring in resected specimens were determined by immunohistochemistry. The number of miR-7-positive cases and the number of cases with higher miR-7 scores were significantly lower among patients with stage I-II NSCLC than those with stage III-IV disease. Additionally, serum levels of miR-7 before and after intervention were lower in stage I-II than in stage III-IV NSCLC cases. Serum levels of miR-7 after treatment were significantly lower than those before intervention in the two groups. The treatment success rate was significantly higher in miR-7-negative patients than in miR-7-positive patients in the two patient groups. Adverse event rates in miR-7-negative and -positive patients were comparable between the groups. Among those with stage III-IV NSCLC, the survival rate of miR-7-positive patients was significantly lower than that of miR-7-negative patients. Conversely, among those with I-II NSCLC, the progression-free survival and median survival time of miR-7-positive patients were significantly lower than those of miR-7-negative patients. Our findings suggest that serum and expression levels of miR-7 in the tissue
\end{abstract}

Correspondence to: Dr Jing Tian, Department of Internal Medicine, Shandong Cancer Hospital Affiliated to Shandong University, Shandong Academy of Medical Sciences, 440 Jiyan Road, Jinan, Shandong 250117, P.R. China

E-mail: tian_jing_111@163.com

Key words: gefitinib, non-small cell lung cancer, miR-7 were closely associated with tumor staging and the therapeutic effects of gefitinib in NSCLC.

\section{Introduction}

Lung cancer accounts for 50-65\% of all cancer diagnoses. Approximately $60-80 \%$ of patients with lung cancer are in advanced stages at diagnosis. For these patients, platinum-based chemotherapy, radiotherapy, and gene-targeted therapy are generally the only treatment options (1). However, the overall efficacy of chemotherapy is only $30-40 \%$, it includes serious side effects, and the overall effective rate over 5 years is only $1-5 \%$ (2).

Gefitinib (Iressa ${ }^{\circledR}$ ), a small molecular-targeted anticancer drug developed by AstraZeneca (New York, NY, USA), is a highly selective oral epidermal growth factor receptor (EGFR) tyrosine kinase inhibitor that was the first of its class to be evaluated in clinical trials. Many large-scale randomized controlled clinical trials demonstrated that gefitinib had better safety and efficacy in controlling renal carcinoma, lung cancer, and moderate and advanced hepatic carcinoma (3-6). Gefitinib, either alone or in combination other chemotherapeutics, is now the first- or second-line treatment option for cancer $(7,8)$.

Recent studies have found that miR-7 was highly expressed in numerous cancers, including breast, lung, liver, and colon cancer (9). Additionally, miR-7 has been shown to play roles in the biological behavior of cancers, including development, migration, and invasion of the tumor, and is a potential target for intervention. Thus, in the present study, we determined the outcomes of gefitinib therapy in patients with non-small cell lung cancer (NSCLC) and its effect on miR-7 expression.

\section{Materials and methods}

Subjects. A total of 126 patients with NSCLC who were admitted to People's Hospital of Laiwu city from January, 2013 to January, 2014, were included in the present study. Histopathology was used to diagnose the stage of cancer in each patient; stage I-II and stage III-IV NSCLC was confirmed in 54 and 72 patients, respectively. Exclusion criteria included concomitant primary cancer in other sites; metastasis to the lung from primary cancer at other sites; comorbidities 
including cardiac, hepatic, pulmonary, kidney, and other organ dysfunction; severe post-surgical complications; advanced disease; expected survival time of less than one month; and incomplete clinical data. The present study was approved by the ethics committee of Shandong Cancer Hospital Affiliated to the Shandong University (Shandong, China). Informed consent was obtained from patients or their legal guardians.

Among patients with stage I-II disease, 22 and 32 patients were diagnosed with stage I and II NSCLC, respectively. Twenty-eight patients were male, with an age range of $38-78(62.4 \pm 15.5)$ years. The maximum diameter of tumor lesions ranged between 0.5 and $4.6(2.6 \pm 1.4) \mathrm{cm}$, and the number of lesions per patient was between 1 and $3(1.4 \pm 0.6)$. In this group, 20 patients underwent open radical tumor resection, and minimally invasive surgery under thoracoscopic guidance was performed in 34 patients. Among patients with stage III-IV cancer, stage III and IV NSCLC was diagnosed in 32 and 40 patients, respectively. Thirty-six patients in this group were male, with an age range of 45-79 (65.0 \pm 17.2$)$ years. The maximum diameter of lesions was $1.4-6.3(3.5 \pm 1.7) \mathrm{cm}$,

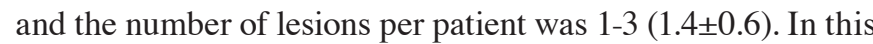
group, liver, bone, lung, and celiac lymph node metastases were found in 16, 15, 3 and 6 patients, respectively.

Clinical approach. Patients with stage I or II NSCLC were treated by surgery in combination with gefitinib chemotherapy, whereas those with stage III or IV NSCLC were treated with gefitinib chemotherapy alone. The same surgical and nursing team attended to all the patients that underwent surgery, according to standard medical procedures. Gefitinib was administered orally at a dose of $250 \mathrm{mg} / \mathrm{day}$, and all the subjects were monitored for any adverse side effects. If necessary, gefitinib treatment was stopped, and patients were observed.

Outcome measures. The effective rate, rate of adverse effects, survival rate, progression-free survival (PFS), and median survival time following treatment were analyzed. Serum miR-7 and tumor positivity for miR-7 before and after surgery in patients with stage I or II NSCLC and before treatment and 3 months after treatment in patients with stage III or IV NSCLC were compared.

According to the response evaluation criteria in solid tumors (RECIST), radiological assessment was conducted once every three months to categorize outcomes as complete remission (CR), partial remission (PR), stable disease (SD), or progressive disease (PD). Specifically, CR was defined as the disappearance of any tumor lesions lasting 4 weeks, and PR was defined as a $>30 \%$ decrease in lesion size lasting 4 weeks. SD was defined as the reduction in lesion size by $<30 \%$ or increase by $\leq 20 \%$ lasting at least 4 weeks, whereas PD was defined as $>20 \%$-increase in lesion size. Effective rate was defined as follows: $\mathrm{CR}+\mathrm{PR}+\mathrm{SD} /$ total number of cases. Acute and chronic toxic and side effects of gefitinib were evaluated and recorded according to the expression and grading standards of the World Health Organization for anticancer drugs (10).

Serum mRNA levels of miR-7 were measured in peripheral mononuclear cells isolated from subjects using reverse transcription-quantitative polymerase chain reaction (RT-PCR) and fluorogenic probes. TRIzol ${ }^{\circledR}$ was used to extract total RNA from the cells, followed by $1.2 \%$ agarose gel electrophoresis and ultraviolet spectrophotometer. The TaqMan ${ }^{\circledR}$ kit was used to determine the quality and concentration of the total RNA. PCR volume was $20 \mu \mathrm{l}$ and included $1 \mu \mathrm{l}$ TaqMan microRNA Assay (x20) probe, $10 \mu 12 \mathrm{X}$ TaqMan general PCR master mix, $7.67 \mu 1$ nuclease-free water, and $1.33 \mu 1$ reverse-transcribed product. The reaction conditions were as follows: $95^{\circ} \mathrm{C}$ for $10 \mathrm{~min}, 95^{\circ} \mathrm{C}$ for $15 \mathrm{sec}, 60^{\circ} \mathrm{C}$ for $1 \mathrm{~min}$; for 40 cycles; 3 replicates were run for each sample. Primers for miR-7 were: forward: 5'-CCAGGACCAGAGGAAACC-3', and reverse: 5'-GCTAGCCTTCTTCTGGATTTGA-3'.

The $2^{-\Delta \Delta \mathrm{Cq}}$ method was used to determine the relative expression of miR-7. Levels of miR-7 expression in resected specimens was determined by immunohistochemistry using previously described steps in the following order: Preparation of paraffin sections, de-waxing, antigen retrieval, endogenous peroxidase activity inhibition, blocking with serum, antibody incubation, 3,3'-diaminobenzidine (DAB) staining, counterstaining, transparency, and mounting.

For the semiquantitative analysis of miR-7 expression, images from five high-power fields were randomly captured, and specimens with $\geq 10 \%$ of tumor cells with positive cytoplasmic miR-7 were determined as positive. Additionally, specimens were scored according to the intensity of cellular miR-7 staining and the number of positive cells. Briefly, specimens with dark (reddish brown) DAB staining and $\geq 50 \%$ immunopositive cells received a score of 3 , specimens with brownish yellow DAB staining and 26-50\% immunopositive cells received a score of 2 , specimens with light yellow DAB staining and $10-25 \%$ immunopositive cells received a score of 1 , and specimens with no visible DAB staining and $<10 \%$ immunopositive cells received a score of 0 .

Statistical analysis. SPSS version 20.0 (IBM SPSS, Armonk, NY, USA) was used for all statistical analyses. Quantitative data were expressed as means \pm standard deviation. Comparisons between groups were determined using the Student's t-test, and within-group comparisons were determined using the paired Student's t-test. Countable data were expressed as numbers with percentages. Groups were compared using Chi-square analysis with Yates' correction, and survival times between groups were determined using the Kaplan-Meier method and log-rank $\chi^{2}$ test. $\mathrm{P}<0.05$ was considered to indicate a statistically significant difference.

\section{Results}

Comparison of tissue and serum miR-7 expression. Immunohistological examination revealed that the number of cases positive for miR-7 and those with higher miR-7 scores were significantly lower in patients with stage I or II NSCLC that were treated with surgery plus gefitinib than those with stage III or IV NSCLC that received only gefitinib chemotherapy (Table I, $\mathrm{P}<0.05$ ). Serum levels of miR-7 in patients with stage I-II NSCLC before and after intervention were significantly lower than those in patients with stage III-IV NSCLC. Furthermore, within-group serum levels of miR-7 after intervention were significantly lower than those before intervention (Table I, $\mathrm{P}<0.05$ ). 
Table I. Comparison of tissue and serum levels of miR-7 expression.

\begin{tabular}{|c|c|c|c|c|c|c|}
\hline \multirow[b]{2}{*}{ Group } & \multirow[b]{2}{*}{ No. of cases } & \multicolumn{3}{|c|}{ Tissue } & \multicolumn{2}{|c|}{ Serum } \\
\hline & & Positive & Negative & Score & Before treatment & After treatment \\
\hline Stage I-II & 54 & $22(40.7)$ & $32(59.3)$ & $1.8 \pm 0.6$ & $0.12 \pm 0.04$ & $0.05 \pm 0.02$ \\
\hline Stage III-IV & 72 & $45(62.5)$ & $27(37.5)$ & $2.6 \pm 0.7$ & $0.46 \pm 0.13$ & $0.27 \pm 0.10$ \\
\hline $\mathrm{t}\left(\chi^{2}\right)$ & & 5.868 & & 6.125 & 6.237 & 6.529 \\
\hline P-value & & 0.015 & & 0.020 & 0.018 & 0.015 \\
\hline
\end{tabular}

Table II. Comparison of treatment success rates and rates of adverse effects (no., \%).

\begin{tabular}{|c|c|c|c|c|c|c|}
\hline Group & No. of cases & CR & PR & SD & PD & Success rate \\
\hline \multicolumn{7}{|l|}{ Stage I-II } \\
\hline Positive & 22 & $3(13.6)$ & $5(22.7)$ & $6(27.3)$ & $8(36.4)$ & $14(63.6)$ \\
\hline Negative & 32 & $9(28.1)$ & $10(31.3)$ & $10(31.3)$ & $3(9.4)$ & $29(90.6)$ \\
\hline$\chi^{2}$ & & & & & & 4.309 \\
\hline P-value & & & & & & 0.038 \\
\hline \multicolumn{7}{|c|}{ Stage III-IV } \\
\hline Positive & 45 & 0 & $3(6.7)$ & $9(20.0)$ & $33(73.3)$ & $12(26.7)$ \\
\hline Negative & 27 & 0 & $6(22.2)$ & $8(29.6)$ & $13(48.1)$ & $14(51.9)$ \\
\hline$\chi^{2}$ & & & & & & 4.639 \\
\hline P-value & & & & & & 0.031 \\
\hline
\end{tabular}

CR, complete remission; $\mathrm{PR}$, partial remission; $\mathrm{SD}$, stable disease; $\mathrm{PD}$, progressive disease.

Table III. Comparison of survival and progression-free survival (PFS) rates and median survival times between early and advanced clinical stages.

\begin{tabular}{|c|c|c|c|c|}
\hline Group & $\begin{array}{l}\text { No. of } \\
\text { cases }\end{array}$ & $\begin{array}{c}\text { Survival rate, } \\
\text { no. }(\%)\end{array}$ & $\begin{array}{l}\text { PFS, } \\
\text { month }\end{array}$ & $\begin{array}{l}\text { Median survival } \\
\text { time, month }\end{array}$ \\
\hline \multicolumn{5}{|l|}{ Stage I-II } \\
\hline Positive & 22 & $15(68.2)$ & 10.3 & 26.8 \\
\hline Negative & 32 & $27(84.4)$ & 14.5 & 31.3 \\
\hline$\chi^{1}$ & & 1.152 & 6.238 & 6.859 \\
\hline $\mathrm{P}$-value & & 0.283 & 0.016 & 0.010 \\
\hline \multicolumn{5}{|c|}{ Stage III-IV } \\
\hline Positive & 45 & $12(26.7)$ & 5.6 & 12.6 \\
\hline Negative & 27 & $14(51.9)$ & 8.4 & 20.2 \\
\hline$\chi^{0}$ & & 4.639 & 6.529 & 7.234 \\
\hline P-value & & 0.031 & 0.013 & 0.008 \\
\hline
\end{tabular}

Comparison of treatment success rates and rates of adverse effects between early and advanced clinical stages. The treatment success rate was higher in miR-7-negative patients than in miR-7-positive patients in stage I-II and III-IV groups; the difference was statistically significant (Table II, P<0.05). The rate of adverse effects was not significantly different between the miR-7-negative and miR-7-positive patients in either stage I-II or stage III-IV NSCLC cases (Table II, P>0.05).
Comparison of survival and PFS rates and median survival time between early and advanced clinical stages. The follow-up time was 23.0-35.0 (30.5 10.4$)$ and 20.0-32.0 $(25.6 \pm 7.4)$ months for patients with stage I-II and III-IV NSCLC, respectively. There were no significant differences in survival rates between miR-7-positive and miR-7-negative patients with stage I or II NSCLC. By contrast, the survival rates were lower in miR-7-positive patients than in miR-7-negative 
patients among those with stage III or IV NSCLC; this difference was statistically significant (Table III, $\mathrm{P}<0.05$ ). The PFS and median survival time were lower in miR-7-positive patients than in miR-7-negative patients among those with stage I or II NSCLC as well as in those with stage III or IV NSCLC. Differences were statistically significant $(\mathrm{P}<0.05)$.

\section{Discussion}

In two double-blind, parallel, multicenter, large-scale, phase II randomized clinical trials, IDEAL1 and IDEAL2 $(3,4)$, gefitinib was used as second-line therapy for advanced NSCLC. The two studies included two groups that received either 250 or $500 \mathrm{mg} /$ day gefitinib. The results of IDEAL1 demonstrated that the objective response rate, symptom improvement rate, and median survival time were 18.4, $43 \%$, and 7.6 months, respectively, in subjects treated with $250 \mathrm{mg} /$ day gefitinib. Conversely, the objective response rate, symptom improvement rate, and median survival time were $19.0,37 \%$, and 8.0 months, respectively, in subjects treated with $500 \mathrm{mg} /$ day gefitinib. The findings of IDEAL2 indicated that the patients receiving $250 \mathrm{mg} /$ day gefitinib achieved an objective response rate of $11.8 \%$ and that the median survival time was 6.1 months, whereas the objective response rate and the medial survival time were $8.8 \%$ and 6.0 months, respectively, in patients treated with $500 \mathrm{mg} /$ day gefitinib.

SIGN, a randomized, phase II clinical trial, was conducted to compare gefitinib with established chemotherapy regimens in Asian populations (5). The results showed that, compared to patients treated with docetaxel, those that received gefitinib as second-line treatment for NSCLC had higher rates of symptom and life quality improvements and objective response rates as well as increased median survival times and fewer drug-related adverse effects. A double-blind, placebo-controlled, multicenter, phase III randomized clinical trial, ISEL, concluded that maximal benefits of gefitinib were observed in patients with adenocarcinoma and those with good PE scores, non-smokers, females, and those carrying EGFR mutations (6). Furthermore, the outcomes of V15-32 (7), INTEREST (8), and ISTANA (9) demonstrated that the efficacy of gefitinib in advanced NSCLC was not inferior to that of docetaxel.

As a randomized phase III clinical trial, IPASS (11) evaluated gefitinib as the first-line treatment for advanced NSCLC and concluded that EGFR mutations were strong predictive biomarkers for the superiority of gefitinib to carboplatin/taxol. Two Japanese trials, WJTOG3405 (12) and NEJGSG002 (13), showed that the PFS of patients with NSCLC and EGFR mutations who were treated with gefitinib was significantly longer than that of patients treated with cisplatin/docetaxel chemotherapy. The Korean First-SIGNAL (14) study also concluded that there were significant differences in PFS rates between gefitinib and platinum-based double-agent chemotherapy as first-line treatment regimen; however, this was not reflected in overall survival rates as the outcomes of the study showed that maximum benefit with gefitinib was observed in patients with EGFR mutations.

Genetic analysis in a randomized phase III trial comparing gefitinib with docetaxel as second-line chemotherapy in NSCLC (7) found that EGFR mutations were present in
40-52.5\% of advanced NSCLC cases and were detectable in $30-40 \%$ of patients with early-stage NSCLC. Moreover, long-term treatment with gefitinib led to the transformation of EGFR mutation-positive tumors to EGFR mutation-negative lesions, which could lead to chemoresistance or weaker chemotherapeutic response and tumor recurrence. Studies have shown that miR-7 expression is closely correlated with the presence and development of lung cancer. Specifically, Xiong et al (15) utilized miR-7 mimics to rescue endogenous miR-7 expression and showed that miR-7 had noticeable inhibitory effects on migration, clone formation, and cell cycle regulation of A549 and H1299 human NSCLC cells in vitro. Conversely, in a nude mouse model, they demonstrated that inhibition of miR-7 gene expression led to the induction of tumor growth and metastasis. Similarly, upregulation of miR-7 may reduce cyclin D1 expression and inhibit the proliferation of NSCLC cell lines.

miR-7 regulates the growth and the metastatic and invasive capabilities of lung cancer cells through multiple mechanisms. Previous tudies showed that miR-7 inhibits the proliferation of 95D lung cancer cells by targeting EGFR $(16,17)$. Xiong et al (15) found that miR-7 targeted the complementary sites of the 3 ' terminal untranslated region of B-cell lymphoma 2 (Bcl-2) to inhibit the expression of $\mathrm{Bcl}-2$, thus inhibiting proliferation and promoting apoptosis in A549 NSCLC cells. They also identified proteasome activator 28 subunit $\gamma$ (PA28 $\gamma)$ as a target of miR-7; miR-7 overexpression led to the downregulation of PA28 $\gamma$ and inhibited the occurrence of NSCLC (17).

In addition to its roles in tumor formation and invasion, miR-7 has been shown to play a role in signal transmission at the plasma membrane in lung cancer cells. Specifically, miR-7 has been shown to negatively regulate the phosphatidylinositol 3-kinase (PI3K)/Akt signaling pathway through the activation of the regulatory subunit of its target protein, PI3K regulatory subunit 3 , which led to the inhibition of growth and migration in lung cancer cells (18). Rai et al (19) transfected miR-7-containing expression vectors into nude mice in an in vivo model of human lung cancer and found that the growth of RPC-9 and H1975 lung cancer cells with high resistance to TKI was significantly inhibited in nude mice bearing tumors, suggesting miR-7 as potential target for gene-targeted therapy in lung cancer.

In this clinical study, we found that the number of miR-7-positive cases and the number of cases with higher levels of miR-7 expression was lower among patients with stage I or II NSCLC than those with stage III or IV cancer. Furthermore, serum levels of miR-7 before and after intervention were lower in stage I-II than in stage III-IV NSCLC cases. Among the early- and advanced-stage patients, serum levels of miR-7 after intervention were lower than those before intervention; these differences in serum levels of miR-7 were statistically significant. The treatment success rate was significantly higher in miR-7-negative patients than in miR-7-positive patients among the early- and advanced-stage patients. The number of adverse effects in miR-7-negative and in miR-7-positive patients was comparable between the early and advanced clinical stage groups. There were no differences in the survival rates of miR-7-positive and miR-7-negative patients with stage I or II NSCLC; however, 
the survival rate of miR-7-positive patients with stage III-IV NSCLC was significantly lower than that of miR-7-negative patients. The PFS and median survival time of miR-7-positive patients with stage I-II NSCLC were significantly lower than those of miR-7-negative patients. In conclusion, serum and tissue miR-7 expression was closely associated with tumor staging and therapeutic effects of gefitinib in NSCLC.

\section{Acknowledgements}

The present study was supported by Science and Technology Planning Project of Shandong Medical Scientific Academy (no. 2014-34).

\section{References}

1. Yung KW, Yung TT, Chung CY, Tong GT, Liu Y, Henderson J, Welbeck D and Oseni S: Principles of cancer staging. Asian Pac J Surg Oncol 1: 1-16, 2015.

2. Zheng YF, Tan LK, Tan BH, Sterling H and Kane R: Principles of surgical oncology. Asian Pac J Surg Oncol 1: 17-26, 2015.

3. Fukuoka M, Yano S, Giaccone G, Tamura T, Nakagawa K, Douillard JY, Nishiwaki Y, Vansteenkiste J, Kudoh S, Rischin D, et al: Multi-institutional randomized phase II trial of gefitinib for previously treated patients with advanced non-small-cell lung cancer (The IDEAL 1 Trial). J Clin Oncol 21: 2237-2246, 2003.

4. Cohen MH, Williams GA, Sridhara R, Chen G and Pazdur R: FDA drug approval summary: Gefitinib (ZD1839) (Iressa) tablets. Oncologist 8: 303-306, 2003.

5. Cufer T, Vrdoljak E, Gaafar R, Erensoy I and Pemberton K; SIGN Study Group: Phase II, open-label, randomized study (SIGN) of single-agent gefitinib (IRESSA) or docetaxel as second-line therapy in patients with advanced (stage IIIb or IV) non-small-cell lung cancer. Anticancer Drugs 17: 401-409, 2006.

6. Thatcher N, Chang A, Parikh P, Rodrigues Pereira J, Ciuleanu T, von Pawel J, Thongprasert S, Tan EH, Pemberton K, Archer V, et al: Gefitinib plus best supportive care in previously treated patients with refractory advanced non-small-cell lung cancer: Results from a randomised, placebo-controlled, multicentre study (Iressa Survival Evaluation in Lung Cancer). Lancet 366: 1527-1537, 2005.

7. Niho S, Ichinose $\mathrm{Y}$, Tamura T, Yamamoto $\mathrm{N}$, Tsuboi $\mathrm{M}$, Nakagawa K, Shinkai T, Jiang H, Nishiwaki Y and Fukuoka M: Results of a randomized Phase III study to compare the overall survival of gefitinib (IRESSA) versus docetaxel in Japanese patients with non-small-cell lung cancer who failed one or two chemotherapy regimens. J Clin Oncol 25, 2007.

8. Kim ES, Hirsh V, Mok T, Socinski MA, Gervais R, Wu YL, Li LY, Watkins CL, Sellers MV, Lowe ES, et al: Gefitinib versus docetaxel in previously treated non-small-cell lung cancer (INTEREST): A randomised phase III trial. Lancet 372: 1809-1818, 2008.
9. Lee DH, Park K, Kim JH, Lee JS, Shin SW, Kang JH, Ahn MJ, Ahn JS, Suh C and Kim SW: Randomized phase III trial of gefitinib versus docetaxel in non-small cell lung cancer patients who have previously received platinum-based chemotherapy. Clin Cancer Res 16: 1307-1314, 2010.

10. Kamil N, Kamil S, Ahmed SP, Ashraf R, Khurram M and Ali MO: Toxic effects of multiple anticancer drugs on skin. Pak J Pharm Sci 23: 7-14, 2010.

11. Yang JC, Wu YL, Chan V, Kurnianda J, Nakagawa K, Saijo N, Fukuoka M, McWalter G, McCormack R and Mok TS: Epidermal grow th factor receptor mutation analysis in previously unanalyzed histology samples and cytology samples from the phase III Iressa Pan-ASia Study (IPASS). Lung Cancer 83: 174-181, 2014.

12. Mitsudomi T, Morita S, Yatabe Y, Negoro S, Okamoto I, Tsurutani J, Seto T, Satouchi M, Tada H, Hirashima T, et al; West Japan Oncology Group: Gefitinib versus cisplatin plus docetaxel in patients with non-small-cell lung cancer harbouring mutations of the epidermal growth factor receptor (WJTOG3405): An open label, randomised phase 3 trial. Lancet Oncol 11: 121-128, 2010.

13. Maemondo M, Inoue A, Kobayashi K, Sugawara S, Oizumi S, Isobe $\mathrm{H}$, Gemma A, Harada M, Yoshizawa H, Kinoshita I, et al; North-East Japan Study Group: Gefitinib or chemotherapy for non-small-cell lung cancer with mutated EGFR. N Engl J Med 362: 2380-2388, 2010.

14. Guetz GD, Landre T, Uzzan B, Chouahnia K, Nicolas P and Morere JF: Is there a survival benefit of first-line epidermal growth factor receptor tyrosine-kinase inhibitor monotherapy versus chemotherapy in patients with advanced non-small-cell lung cancer?: A meta-analysis. Target Oncol 11: 41-47, 2016.

15. Xiong S, Zheng Y, Jiang P, Liu R, Liu X, Qian J, Gu J, Chang L, Ge D and Chu Y: PA28gamma emerges as a novel functional target of tumour suppressor microRNA-7 in non-small-cell lung cancer. Br J Cancer 110: 353-362, 2014.

16. Hu Y, Liao Z, Chen C, Qin N, Zheng J, Tian D, Li Y, Zhu S, Luo J and Xu L: Over-expressed microRNA-7 inhibits the growth of human lung cancer cells via suppressing CGGBP1 expression. Xi Bao Yu Fen Zi Mian Yi Xue Za Zhi 30: 125-130, 2014 (In Chinese).

17. Zhang $\mathrm{Z}$ and Zhang R: Proteasome activator PA28 gamma regulates $\mathrm{p} 53$ by enhancing its MDM2-mediated degradation. EMBO J 27: 852-864, 2008.

18. Xu L, Wen Z, Zhou Y, Liu Z, Li Q, Fei G, Luo J and Ren T: MicroRNA-7-regulated TLR9 signaling-enhanced growth and metastatic potential of human lung cancer cells by altering the phosphoinositide-3-kinase, regulatory subunit 3/Akt pathway. Mol Biol Cell 24: 42-55, 2013.

19. Rai K, Takigawa N, Ito S, Kashihara H, Ichihara E, Yasuda T, Shimizu K, Tanimoto M and Kiura K: Liposomal delivery of MicroRNA-7-expressing plasmid overcomes epidermal growth factor receptor tyrosine kinase inhibitor-resistance in lung cancer cells. Mol Cancer Ther 10: 1720-1727, 2011. 